\title{
Lung segmentectomy assisted by highly selective independent segmental ventilation: a series of three cases
}

Xiaoshun Shi ${ }^{1+}$, Jing $\mathrm{Ye}^{2 \dagger}$, Junyong Chen ${ }^{2}$, Jianxue Zhai ${ }^{1}$, Xiguang $\mathrm{Liu}^{1}$, Di Lu${ }^{1}$, Zishi $\mathrm{Lin}^{2}, \mathrm{Zhen} \mathrm{Ni}^{1}, \mathrm{Hua} \mathrm{Wu}^{1}$ and Kaican $\mathrm{Cai}^{1 *}$ (D)

\begin{abstract}
Background: The identification of targeted intersegmental planes and resection with adequate surgical margins are among the crucial steps in anatomical pulmonary segmentectomy, and technical improvements are still needed.

Case presentation: We reported three cases of intersegmental plane identification using highly selective independent segmental ventilation during segmentectomy. All cases required cooperation with an anesthesiologist who was able to perform segmental ventilation and double confirmation of segmental bronchus branches by the surgeon. The surgical procedure provides a direct visualization of spare segment inflation and saves time in deflation over the conventional residual segment inflation method.
\end{abstract}

Conclusions: Highly selective independent segmental ventilation could be considered a suitable option for pulmonary intersegmental plane identification and could be universally used for lung segmentectomy.

Keywords: Segmentectomy, Highly selective independent segmental ventilation, VATS, Intersegmental plane identification

\section{Background}

Precise intersegmental plane identification is a crucial step in anatomical segmentectomy, which is a surgical procedure aimed at curing small and early lung cancer while preserving lung function. Previous approaches have mainly focused on the modification of medical dyes and ventilation techniques, such as direct injection of indocyanine green (ICG) [1] and methylene blue [2] into the bronchi of target pulmonary segments, indirect intravenous injection of ICG visualized by infrared thoracoscopy (IRT) [3], inflation-deflation methods [4], the selective jet injection method [5], oxygen instillation into affected segments with a butterfly needle [6], the slip knot bronchial ligation method, and the modified inflation-

\footnotetext{
*Correspondence: doc_cai@163.com

${ }^{+}$Xiaoshun Shi and Jing Ye contributed equally to this work.

'Department of Thoracic Surgery, Nanfang Hospital, Southern Medical University, No. 1838 North Guangzhou Avenue, Guangzhou 510515, China Full list of author information is available at the end of the article
}

deflation method [7]. These techniques are demanding due to time for lung collapse, pharmaceutical materials, special cameras, and potential risk of air embolism [8]. Therefore, a simpler and efficient method of intersegmental plane identification is required. With the advancement of the anesthesiologic technique, one-lung ventilation is possible [9], reaching the segmental level [10]. With specialized instruments and proper intubation techniques, direct inflation of a lung segment is possible. We reported three successful cases of intersegmental plane identification assisted using highly selective independent segmental ventilation during thoracoscopic segmentectomy.

\section{Case presentation}

\section{Patients and surgical procedures}

Three patients, 52, 78, and 60 years old, were admitted because small nodules less than $3 \mathrm{~cm}$ in size were found 
on computed tomography between April 2018 and May 2018. All of the patients met the indications for pulmonary segmentectomy. The VATS procedures included the lateral basal and posterior basal segments of the right lower lung (Case 1: S9+10), the apical, posterior and anterior segments of the left upper lobe (Case 2: $\mathrm{S} 1+2+3)$, and the apical and posterior segments of the left upper lobe (Case 3: $\mathrm{S} 1+2)$. All segmentectomies were performed by single-direction thoracoscopic segmentectomy [11-14].

\section{Highly selective independent segmental ventilation}

The ventilation technique was approved by the Institutional Review Board and the Ethics Committee of Nanfang Hospital, Southern Medical University (IRB- 2012156). A schematic representation of highly selective independent segmental ventilation is shown in Fig. 1.

\section{Preparation}

Before anesthesia and ventilation, all of the patients were excluded from tracheal and bronchial variation by assessing the results of preoperative chest computed tomography and bronchoscopy. Anesthesia induction was performed by propofol sedation using target-controlled infusion, and then the use of a double-lumen endobronchial tube (DLT) was based on the bronchial diameter at the level of the sternal end of the clavicle. In general, we used 37 French gauges ( $\mathrm{Fr}$ ) with internal tube diameters (ITDs) of $4.7 \mathrm{~mm}$ or larger in most male patients and 32
Fr with ITDs of $3.5 \mathrm{~mm}$ or larger in most female patients. The use of the 5F Arndt pediatric endobronchial blocker (Cook Medical, Bloomington, USA) was based on product instructions. Notably, upon the completion of segment ventilation, the balloon endobronchial blocker must be deflated before removal.

\section{Step 1. Identification of independent segmental bronchial orifice}

Two experienced anesthesiologists identified segmental bronchial orifices by fiberoptic bronchoscopy (FOB), advancing the endobronchial blocker to the orifice of independent segmental bronchus.

\section{Step 2. Intraoperative conformation of independent segmental bronchial orifice}

Following the management of the target segmental structure (segmental vein, artery, and bronchus), intersegmental plane identification is required. Currently, the 5F Arndt pediatric endobronchial blocker enters the initial placement guided by $\mathrm{FOB}$ and is inserted into the orifice of the segmental bronchus of the independentventilated pulmonary segment (Fig. 2a-b).

\section{Step 3. Ventilation of independent pulmonary segment}

After reconfirmation by the light of FOB under VATS dark vision (Fig. 2c) and the endo-stapler being clamped at the targeted segmental bronchi, 100\% oxygen flowing at $3-5 \mathrm{~L} / \mathrm{min}$ was ventilated by an anesthesiologist (Fig.

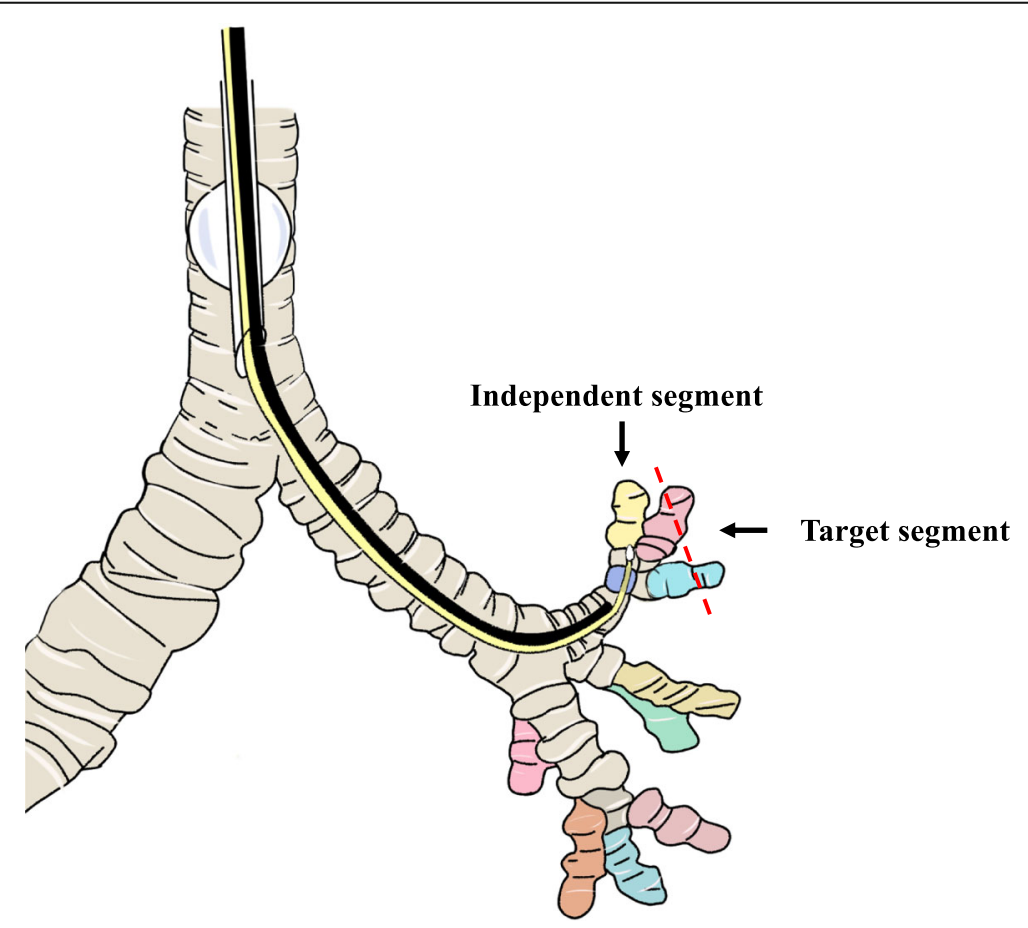

Fig. 1 Schematic representation of highly selective independent segmental ventilation 


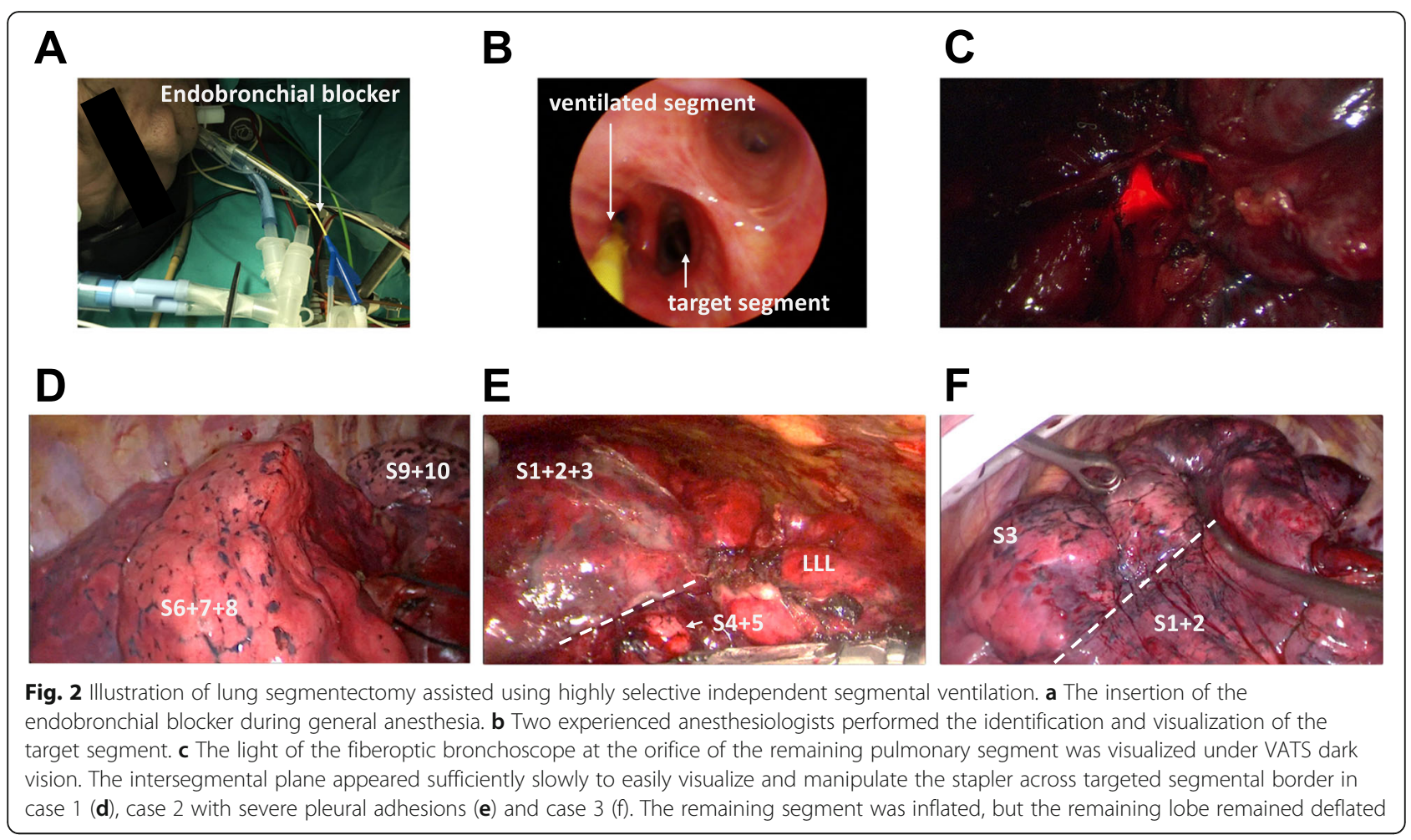

$2 d-f)$. The independent pulmonary segments were subsequently inflated without ventilation of the remaining pulmonary lobe.

After triple confirmation and marking of the intersegmental plane, an endo-stapler (Endo GIA ${ }^{\mathrm{ma}} 60 \mathrm{~mm}$ Articulating Medium/Thick Reload with Tri-Staple Technology Purple Cartridge, COVIDIEN Medical, USA) was introduced, and the segmental bronchi and the segmental border were divided. Lymph node dissection was then performed.

\section{Discussion and conclusions}

We successfully performed 3 segmentectomies with highly selective independent segmental ventilation. There were no ventilation-associated complications after surgery. No segmentectomy-caused complications, such as air leaks, atelectasis or subcutaneous emphysema, were observed. The patients were discharged with an uneventful recovery course. A technique of quick and accurate identification of the plane of the lung segment is of particular importance in segmental resection. The conventional method is the inflation and deflation technique. The shortcoming of this method is that the identification of the target bronchus depends greatly on the subjective judgment of the surgeon, and the waiting time for deflation is long. Other identification techniques for the intersegmental plane have been described in previous studies. The technique of ultrafine fiber bronchoscopy with high-frequency bronchial ventilation $(40 \mathrm{~Hz}, 2$ $\mathrm{kg} / \mathrm{cm} 2)$ at the target segment was proposed by Okada
[15]. Misaki et al. visualized intersegmental junctions under infrared thoracoscopy when intravenous injection of indocyanine green (ICG; $3.0 \mathrm{mg} / \mathrm{kg}$ ) was performed after ligation of the target segmental artery. Oh et al. reported a method of injection of ICG ( $25 \mathrm{mg}$ dissolved in $50 \mathrm{~mL}$ of saline) at the distal peripheral bronchus after the target segment bronchus was controlled [16].

Highly selective lung segment ventilation is an anesthetic-surgical technique that combines the proficiency of bronchoscopy, ventilation and thoracoscopy under close cooperation between the surgeon and the anesthesiologist. The trinity of segmental bronchus identification by bronchoscopy, bronchoscopy light source under dark vision using VATS, and inflation of the remaining segment not affecting other pulmonary lobes ensures the safety of precise resection of the target lung segment. Compared with traditional ventilation, our highly selective independent segmental ventilation does not ventilate the remaining pulmonary lobe, avoiding the waiting time for lung collapse and not blocking the surgical field. Compared with traditional bronchoscopeguided high-frequency ventilation, our endobronchial blocker was inserted into the orifice of the independent segmental bronchus, which could effectively prevent potential damage to the endobronchial blocker and the bronchoscope. Therefore, this technology can accurately and quickly locate the stereoborder of the target lung segment, which is expected to shorten the operative time for lung segment resection and improve the efficiency of intersegmental plane identification. 
The disadvantages of this technique include incurring the additional cost of the endobronchial blocker and the anesthesiologist's experience in bronchoscopy beyond the trachea and main bronchus. However, the endobronchial blocker used in this technique is a conventional consumable. No costly special thoracoscopic equipment or dye injection is required. From anesthesiologists' point of view, the idea of selective lobar blockade, selective segmental blockade or ventilation lives on in new, adaptive forms. With the increasing demand for ERAS in thoracic surgery, experienced anesthesiologists use this novel concept of lung isolation, which benefits our patients. Several different available bronchial blockers can be used to achieve lobar or segmental collapse; therefore, understanding the bronchoscopic anatomy of the pulmonary segment should be assured and is required in our hospital.

To our knowledge, this study is the first case series of pulmonary segmentectomy assisted by highly selective independent segmental ventilation. Because of the simplicity of this technique, we purpose that universal use could be feasible for resection of pulmonary segments, and the efficacy of this method should be estimated in a larger clinical trial.

\section{Abbreviations}

DLT: Double-lumen endobronchial tube; FOB: Fiberoptic bronchoscopy; Fr: French gauges; ICG: Indocyanine green; IRT: Infrared thoracoscopy; ITD: Internal tube diameter; VATS: Video-assisted thoracoscopic surgery

\section{Acknowledgments}

Refer to the Funding section.

\section{Authors' contributions}

KC C and XS S suggested the method and drafted the manuscript. KC C, XS S, $X G L, D L, Z N$, and $H W$ performed the surgeries. J $Y$ and JY $C$ performed the anesthesia procedures. XS S, JX Z, ZS L and Z N contributed to editing and revising the manuscript. All of the authors read and approved the final manuscript.

\section{Funding}

The work was supported by the Science and Technology Planning Project of Guangdong Province (2016A020215120), the Research Initiative Fund of Southern Hospital 2018 (C1051325), and the National Natural Science Foundation of China (No. 81902319). The work was partially supported by the Major Science and Technology Planning Project of Guangdong Province (2017B020226005) and the National Natural Science Foundation of China (81902319).

\section{Availability of data and materials}

All images supporting the technique of this article are included within the article. We could provide surgical videos with patient consent.

\section{Declarations}

\section{Ethics approval and consent to participate}

This ventilation technique was approved by the Institutional Review Board and the Ethics Committee of Nanfang Hospital, Southern Medical University. Written informed consent was obtained from the patients for publication of this technical procedure.

\section{Consent for publication}

Consent for publication was obtained from the presented patients.

\section{Competing interests}

We declare no competing interests.

\section{Author details}

${ }^{1}$ Department of Thoracic Surgery, Nanfang Hospital, Southern Medical University, No. 1838 North Guangzhou Avenue, Guangzhou 510515, China. ${ }^{2}$ Department of Anesthesiology, Nanfang Hospital, Southern Medical University, Guangzhou 510515, China.

Received: 29 September 2020 Accepted: 5 April 2021

Published online: 15 April 2021

\section{References}

1. Sekine $Y$, Ko E, Oishi $H$, Miwa M. A simple and effective technique for identification of intersegmental planes by infrared thoracoscopy after transbronchial injection of indocyanine green. J Thorac Cardiovasc Surg. 2012;143(6):1330-5. https://doi.org/10.1016/j.jtcvs.2012.01.079.

2. Zhang Z, Liao Y, Ai B, Liu C. Methylene blue staining: a new technique for identifying intersegmental planes in anatomic segmentectomy. Ann Thorac Surg. 2015;99(1):238-42. https://doi.org/10.1016/j.athoracsur.2014.07.071.

3. Misaki N, Chang SS, Igai H, Tarumi S, Gotoh M, Yokomise H. New clinically applicable method for visualizing adjacent lung segments using an infrared thoracoscopy system. J Thorac Cardiovasc Surg. 2010;140(4):752-6. https://doi.org/10.1016/j.jtcvs.2010.07.020.

4. Churchill ED, Belsey R. Segmental Pneumonectomy in bronchiectasis: the Lingula segment of the left upper lobe. Ann Surg. 1939;109(4):481-99. https://doi.org/10.1097/00000658-193904000-00001.

5. Matsuoka H, Nishio W, Sakamoto T, Harada H, Yoshimura M, Tsubota N. Selective segmental jet injection to distinguish the intersegmental plane using jet ventilation. Jpn J Thorac Cardiovasc Surg. 2003;51(8):400-1. https:// doi.org/10.1007/BF02719478.

6. Kamiyoshihara M, Kakegawa S, Morishita Y. Convenient and improved method to distinguish the intersegmental plane in pulmonary segmentectomy using a butterfly needle. Ann Thorac Surg. 2007;83(5):19134. https://doi.org/10.1016/j.athoracsur.2006.06.052.

7. Wang J, Xu X, Wen W, Wu W, Zhu Q, Chen L. Modified method for distinguishing the intersegmental border for lung segmentectomy. Thorac Cancer. 2018;9(2):330-3. https://doi.org/10.1111/1759-7714.12540.

8. Otsuka T, Nakamura Y, Harada A, Sato M. Extremely rare but potential complication of diffuse brain edema due to air embolism during lung segmentectomy with selected segmental inflation technique by syringe needle during video-assisted thoracoscopic surgery. J Thorac Cardiovasc Surg. 2011;142(5):e151-2. https://doi.org/10.1016/j.jtcvs.2011.07.061.

9. Knoll H, Ziegeler S, Schreiber JU, Buchinger H, Bialas P, Semyonov K, et al. Airway injuries after one-lung ventilation: a comparison between double-lumen tube and endobronchial blocker: a randomized, prospective, controlled trial. Anesthesiology. 2006;105(3):471-7. https://doi.org/10.1097/00000542-200609000-00009.

10. Amar D, Desiderio DP, Bains MS, Wilson RS. A novel method of one-lung isolation using a double endobronchial blocker technique. Anesthesiology. 2001;95(6):1528-30. https://doi.org/10.1097/00000542-200112000-00039.

11. Liu L, Che G, Pu Q, Ma L, Wu Y, Kan Q, et al. A new concept of endoscopic lung cancer resection: single-direction thoracoscopic lobectomy. Surg Oncol. 2010;19(2):e71-7. https://doi.org/10.1016/j.suronc.2009.04.005.

12. Liu C, Guo C, Lin F, Li C, Pu Q, Ma L, et al. Long term outcomes of patients with stage I lung cancer treated by single-direction video-assisted thoracoscopic surgery lobectomy or segmentectomy. Zhonghua Wai Ke Za Zhi. 2015;53(10):742-6.

13. Cai $K$, Wu H, Ren $P$, Cai $R$, Xiong G, Wang H. Unidirectionally progressive resection of lower right lung cancer under video-assisted thoracoscopy. J Thorac Dis. 2013:5(Suppl 3):S310-4.

14. Liu C, Liao H, Guo C, Pu Q, Mei J, Liu L. Single-direction thoracoscopic basal segmentectomy. J Thorac Cardiovasc Surg. 2020;160(6):1586-94. https://doi. org/10.1016/j.jtcvs.2020.01.028.

15. Okada M, Koike T, Higashiyama M, Yamato Y, Kodama K, Tsubota N. Radical sublobar resection for small-sized non-small cell lung cancer: a multicenter study. J Thorac Cardiovasc Surg. 2006;132(4):769-75. https:/doi.org/10.1016/j.jtcvs.2006.02.063.

16. Oh S, Suzuki K, Miyasaka Y, Matsunaga T, Tsushima Y, Takamochi K. New technique for lung segmentectomy using indocyanine green injection. Ann Thorac Surg. 2013; 95(6):2188-90. https://doi.org/10.1016/j.athoracsur.2012.12.068.

\section{Publisher's Note}

Springer Nature remains neutral with regard to jurisdictional claims in published maps and institutional affiliations. 